\title{
Pressure Dependence of the Light Emission in Zinc-Blende InGaAs/GaAs and InGaN/GaN Quantum Wells
}

\author{
S.P. ŁEPKOWSKI AND I. GORCZYCA \\ Unipress - Institute of High Pressure Physics, Polish Academy of Sciences \\ Sokołowska 29, 01-142 Warszawa, Poland
}

\begin{abstract}
We present theoretical study of the pressure coefficient of the light emission $\left(\mathrm{d} E_{\mathrm{E}} / \mathrm{d} P\right)$ in compressively strained zinc-blende InGaAs/GaAs and InGaN/GaN quantum wells, grown in a (001) direction. We investigate the contributions to $\mathrm{d} E_{\mathrm{E}} / \mathrm{d} P$ arising from (i) third-order (nonlinear) elasticity, (ii) nonlinear elasticity, originating from pressure dependence of elastic constants, and (iii) nonlinear dependence of elastic constants on composition in InGaAs and InGaN alloys. The obtained results indicate that the use of nonlinear elasticity is essential for determination of $\mathrm{d} E_{\mathrm{E}} / \mathrm{d} P$ in the strained InGaAs/GaAs and InGaN/GaN quantum wells, while the inclusion of the nonlinear dependence of elastic constants on composition of InGaAs and InGaN alloys does not improve agreement between the theoretical end experimental values of $\mathrm{d} E_{\mathrm{E}} / \mathrm{d} P$ in the considered structures.
\end{abstract}

PACS numbers: 62.20.D-, 62.50.-p, 81.40.Jj, 78.67.De

\section{Introduction}

Pressure dependence of the light emission in semiconductor quantum wells (QWs) has been extensively studied in the recent years [1-5]. These studies have revealed that pressure coefficient of the light emission $\left(\mathrm{d} E_{\mathrm{E}} / \mathrm{d} P\right)$ for a QW can be substantially different from the pressure coefficient of the band gap for unstrained bulk material of the same chemical composition. It has been discovered that the magnitude of $\mathrm{d} E_{\mathrm{E}} / \mathrm{d} P$ in QWs depends significantly on the built-in biaxial strains and piezoelectric fields. Unexpectedly, the linear theory of elasticity and piezoelectricity often failed to predict the values of $\mathrm{d} E_{\mathrm{E}} / \mathrm{d} P$ in semiconductor QWs, and the nonlinear elastic and piezoelectric effects have been taken into account [1-4].

In this work, we present the results of theoretical study of $\mathrm{d} E_{\mathrm{E}} / \mathrm{d} P$ in compressively strained zinc-blende InGaAs/GaAs and InGaN/GaN QWs, grown in a (001) direction. In these structures the built-in electric field is not present but the magnitude of $\mathrm{d} E_{\mathrm{E}} / \mathrm{d} P$ is significantly reduced by the presence of the built-in compressive biaxial strain originating from the lattice misfit between barriers and QWs $[1,4]$. The linear theory of elasticity with linear dependence of the second-order elastic constants $\left(C_{i j}\right)$ on alloy composition failed to predict this effect $[1,4]$. Therefore, we investigate the contributions to $\mathrm{d} E_{\mathrm{E}} / \mathrm{d} P$ arising from (i) third-order (nonlinear) elasticity, (ii) nonlinear elasticity, originating from pressure dependence of $C_{i j}$, (iii) nonlinear dependence of $C_{i j}$ on composition in InGaAs and InGaN alloys.

\section{Models}

In order to study the contributions to $\mathrm{d} E_{\mathrm{E}} / \mathrm{d} P$ originating from the effects (i) and (ii), we have used two

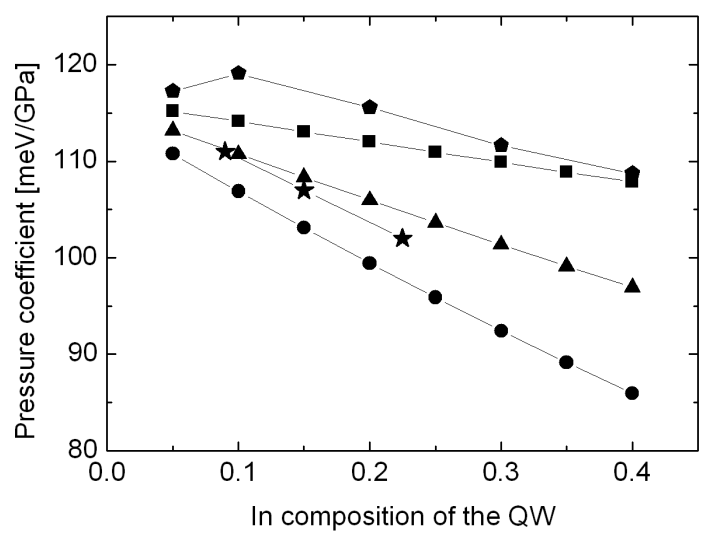

Fig. 1. The values of $\mathrm{d} E_{\mathrm{E}} / \mathrm{d} P$ for $10 \mathrm{~nm}$ InGaAs/GaAs QWs, as a function of In concentration. Stars represent the experimental results (taken from Ref. [1]), squares, triangles, circles, and pentagons correspond to the theoretical results which are obtained using (i) the linear theory of elasticity, (ii) the third-order elasticity theory, (iii) nonlinear elasticity originating from the pressure dependence of the second-order elastic constants, and (iv) the linear theory of elasticity with nonlinear dependence of elastic constants on composition of InGaAs alloys, respectively. Solid lines are added to guide the eye.

models of pressure-tuning of strains in (001)-oriented zinc-blende QWs, which have been presented in detail in Ref. [4]. Using these models, we have calculated the values of $\mathrm{d} E_{\mathrm{E}} / \mathrm{d} P$ for strained InGaAs/GaAs and InGaN/GaN QWs, assuming linear dependence of the second-order $\left(C_{i j}\right)$ and third-order $\left(C_{i j k}\right)$ elastic constants on In content in the QWs [4].

To investigate the contribution to $\mathrm{d} E_{\mathrm{E}} / \mathrm{d} P$ originating from the effect (iii), we have performed ab initio calcula- 


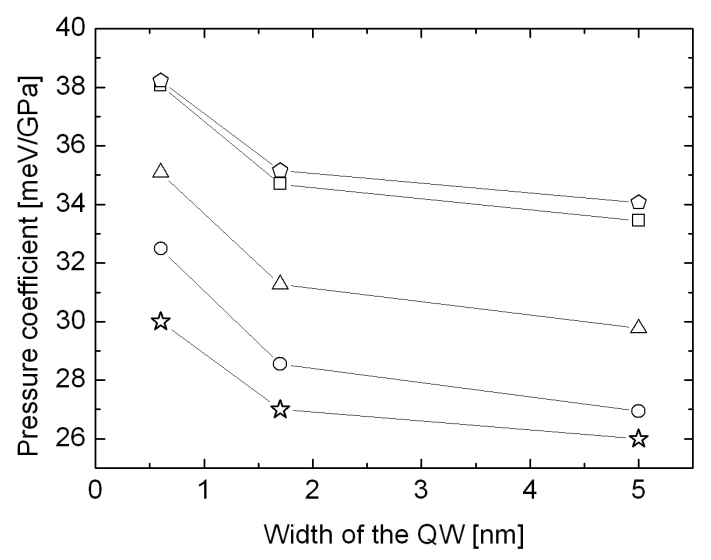

Fig. 2. The values of $\mathrm{d} E_{\mathrm{E}} / \mathrm{d} P$ for zinc-blende $\mathrm{In}_{0.1} \mathrm{Ga}_{0.9} \mathrm{~N} / \mathrm{GaN} \mathrm{QWs}$, as a function of $\mathrm{QW}$ width. Stars represent the experimental results taken from Ref. [5], squares, triangles, circles, and pentagons correspond to the theoretical results which are obtained using (i) the linear theory of elasticity, (ii) the third-order elasticity theory, (iii) nonlinear elasticity originating from the pressure dependence of the second-order elastic constants, and (iv) the linear theory of elasticity with nonlinear dependence of elastic constants on composition of InGaN alloys, respectively. Solid lines are added to guide the eye.

tions of elastic constants $C_{i j}$ in zinc-blende InGaN alloys. Slightly sub-linear dependence of $C_{i j}$ on In content has been found. In the case of InGaAs alloys, the elastic constants $C_{i j}$ also depend sub-linearly on composition [6]. Then, we have calculated the values of $\mathrm{d} E_{\mathrm{E}} / \mathrm{d} P$ in the InGaN/GaN and InGaAs/GaAs QWs, assuming linear and sub-linear dependences of $C_{i j}$ on composition of In$\mathrm{GaN}$ and InGaAs alloys.

\section{Results}

The obtained results are shown in Figs. 1 and 2. One can see that the use of third-order elasticity leads to significant reduction of $\mathrm{d} E_{\mathrm{E}} / \mathrm{d} P$ in both InGaAs/GaAs and $\mathrm{InGaN} / \mathrm{GaN}$ QWs, in comparison to the values of $\mathrm{d} E_{\mathrm{E}} / \mathrm{d} P$ obtained by using the linear theory of elasticity [4]. In the case of InGaAs/GaAs QWs, the values of $\mathrm{d} E_{\mathrm{E}} / \mathrm{d} P$ calculated using third-order elasticity are in reasonable agreement with experimental data taken from Ref. [1]. For zinc-blende InGaN/GaN QWs, bet- ter agreement between theoretical and experimental values (taken from Ref. [5]) of $\mathrm{d} E_{\mathrm{E}} / \mathrm{d} P$ is obtained when instead of third-order elasticity, pressure dependence of $C_{i j}$ is taken into account [4]. On the other hand, we have found that the use of the sub-linear dependence of $C_{i j}$ on composition of InGaN alloys leads to small increase of the $\mathrm{d} E_{\mathrm{E}} / \mathrm{d} P$ in InGaN/GaN QWs, in comparison to the values of $\mathrm{d} E_{\mathrm{E}} / \mathrm{d} P$ obtained assuming linear dependence of $C_{i j}$ on In content. Similar effect is found for $\mathrm{d} E_{\mathrm{E}} / \mathrm{d} P$ in InGaAs/GaAs QWs.

\section{Conclusion}

In conclusion, we have investigated the contributions to $\mathrm{d} E_{\mathrm{E}} / \mathrm{d} P$ in strained InGaAs/GaAs and InGaN/GaN QWs arising from the nonlinear elasticity effects and nonlinear dependence of $C_{i j}$ on composition in InGaAs and InGaN alloys. The obtained results indicate that the use of nonlinear elasticity is essential for determination of the values of $\mathrm{d} E_{\mathrm{E}} / \mathrm{d} P$ in the compressively strained QWs. The inclusion of the nonlinear dependence of $C_{i j}$ on composition of InGaAs and InGaN alloys does not improve agreement between the theoretical end experimental values of $\mathrm{d} E_{\mathrm{E}} / \mathrm{d} P$ in the considered structures.

\section{Acknowledgments}

This work was supported by the Polish State Committee for Scientific Research, project No. NN202 010134.

\section{References}

[1] V.A. Wilkinson, A.D. Prins, J.D. Lambkin, E.P. O'Reilly, D.J. Dunstan, L.K. Howard, M.T. Emeny, Phys. Rev. B 42, 3113 (1990); M.D. Frogley, J.R. Downes, D.J. Dunstan, Phys. Rev. B 62, 13612 (2000).

[2] G. Vaschenko, D. Patel, C.S. Menoni, N.F. Gardner, J. Sun, W. Gotz, C.N. Tome, B. Clausen, Phys. Rev. B 64, 241308(R) (2001).

[3] S.P. Łepkowski, Phys. Rev. B 77, 155327 (2008).

[4] S.P. Łepkowski, Phys. Rev. B 78, 153307 (2008).

[5] T. Suski, H. Teisseyre, S.P. Łepkowski, P. Perlin, T. Kitamura, Y. Ishida, H. Okumura, S.F. Chichibu, Appl. Phys. Lett. 81, 232 (2002).

[6] P.S. Branicio, J.P. Rino, F. Shimojo, R.K. Kalia, A. Nakano, P. Vashishta, J. Appl. Phys. 94, 3840 (2003). 